\title{
Transrectal ultrasound biopsy of the prostate: does it still have a role in prostate cancer diagnosis?
}

\author{
Andrew Moe ${ }^{1,2,3}$, Dickon Hayne $e^{1,2,3}$ \\ ${ }^{1}$ Department of Urology, Fiona Stanley Hospital, Murdoch, Western Australia, Australia; ${ }^{2}$ UWA Medical School, University of Western Australia, \\ Crawley, Western Australia, Australia; ${ }^{3}$ Australian and New Zealand Urogenital and Prostate Cancer Trials Group (ANZUP), Sydney, New South \\ Wales, Australia \\ Contributions: (I) Conception and design: All authors; (II) Administrative support: None; (III) Provision of study materials or patients: None; (IV) \\ Collection and assembly of data: All authors; (V) Data analysis and interpretation: All authors; (VI) Manuscript writing: All authors; (VII) Final \\ approval of manuscript: All authors. \\ Correspondence to: Dickon Hayne. Department of Urology, Fiona Stanley Hospital, Murdoch, Western Australia, Australia. Email: dickon.hayne@uwa.edu.au.
}

\begin{abstract}
Transrectal ultrasound (TRUS) guided biopsy of the prostate has been a standard diagnostic approach for prostate cancer over the past thirty years. Today, the role of TRUS biopsy is being challenged by transperineal (TP) prostate biopsy due to concerns over the safety and diagnostic yield of TRUS biopsy. TRUS biopsy still offers a convenient, reliable and accessible tool for diagnosing prostate cancer in the majority of patients. It continues to play a role in prostate cancer diagnosis, especially where hospital resource allocation is limited, including the public sector. TRUS biopsy has low rates of severe complications, although there remains room for improvement in current practice to improve the tolerability and reduce the incidence of post-biopsy infection.
\end{abstract}

Keywords: Transrectal ultrasound (TRUS); biopsy; prostate cancer

Submitted May 31, 2019. Accepted for publication Sep 04, 2019.

doi: 10.21037/tau.2019.09.37

View this article at: http://dx.doi.org/10.21037/tau.2019.09.37

\section{Introduction}

Transrectal ultrasound (TRUS) guided biopsy of the prostate is performed to obtain a histopathological diagnosis of prostate cancer and has been a mainstay of urological practice for almost thirty years. In that time, the techniques involved have been refined to improve the detection rate of malignancy, to better estimate tumour burden and to assist in surgical planning. Similarly, improvements in perioperative care have enhanced tolerability and reduced the associated sepsis rates complicating this biopsy technique.

A review of TRUS biopsy is timely as technology advances. An increasing body of evidence has emerged in support of transperineal (TP) biopsy as the preferred technique for pathological sampling of the prostate gland; however, TRUS biopsy remains accessible and widely practiced, and is likely to have a role in urological practice for some time.

This review addresses the history and development of TRUS biopsy, refinements in technique and clinical practice, the advantages and disadvantages of TRUS when compared to TP biopsy, and the evidence surrounding TRUS biopsy in today's practice.

\section{A history of TRUS guided biopsy of the prostate}

The first transrectal biopsy of the prostate was described by Astraldi in 1937 (1). The anatomical position of the prostate lends itself to easy palpation and thus superficial biopsy techniques were feasible. TRUS was first applied by Takahashi and Ouchi in 1964, and was further refined by Watanabe and colleagues in 1967 (2). As technology evolved, abnormalities associated with locally advanced prostate adenocarcinoma were soon detectable using TRUS (2). 


\section{The first modern TRUS biopsy}

In 1989, Hodge and colleagues described the first clinically useful TRUS guided prostate biopsy. This was achieved by aligning palpable abnormalities and sonographic findings to guide biopsy (3). Hodge subsequently described the sextant technique for systematic random biopsy of the prostate. Using a spring-loaded biopsy gun, mapped biopsies were taken from the bilateral prostatic lobes at the apex, middle and base. Hodge proposed that this could be a useful aid in estimating the tumour grade, stage and surgically relevant anatomical location, as compared to targeted biopsy of abnormal prostatic lesions alone (4). It subsequently became the standard diagnostic approach for prostate biopsy.

\section{Refining the technique}

As TRUS biopsy entered standard clinical practice, clinicians began to correlate biopsy findings to the overall tumour burden. Dietrick and colleagues proposed that tumour volume could be linked to the size of malignant tissue in the TRUS biopsy core, and that this information could guide cancer management (5). Reliable prediction of tumour volume required improvements to the traditional sextant technique, and the optimal location and number of biopsies was a source of published debate. Variants of this method have been adopted in different centres to improve diagnostic yield. These modifications focused on increasing the number of cores taken while targeting the lateral aspect of the prostate to better sample the peripheral zone (6). Additional lateral biopsies were also employed in larger prostates (7). These variant biopsy techniques improved prostate cancer detection rates by a range of $20-35 \%$ (6-9), and have been widely adopted in Urological practice.

\section{TRUS biopsy technique-an example}

A standard technique as performed in our institute is described as follows. TRUS biopsy of the prostate is performed as a day case. The patient receives $500 \mathrm{mg}$ of oral ciprofloxacin one hour before the procedure. The urologist is positioned to the right of the supine patient. The patient is then moved into the left lateral decubitus position to commence the procedure.

Digital rectal examination is performed, and findings are recorded. A Bard spring-loaded biopsy gun and a $7 \mathrm{MHz}$ endorectal biplanar ultrasound probe are used. The probe is covered with ultrasound jelly, a thin plastic sheath, and a repeat coat of jelly. The probe is inserted into the rectum and is directed toward the anterior wall, where the prostate can be visualised. Five millilitre of $1 \%$ lidocaine is injected into the peri-prostatic neurovascular bundles on each side of the basolateral aspects of the prostate. The prostate volume is then measured, before 14 systematic core prostate biopsies are obtained. Two cores are taken from both sides of the base, mid, and apex of the prostate. Bilateral samples are obtained from the transition zone.

\section{Complications}

TRUS biopsy is an overall well tolerated procedure. When surveyed, over $80 \%$ of patients who underwent TRUS biopsy in our centre would have a repeat procedure under local anaesthetic as required (10). TRUS biopsy is associated with some risks including pain, acute urinary retention, haematuria, haematospermia, rectal bleeding, erectile dysfunction, infection and sepsis (11). The rising rates of infection and sepsis internationally are of concern and are discussed in more detail below. The relative measure of acute urinary retention post biopsy is also reviewed.

\section{Comparing TRUS biopsy against TP biopsy}

The advantages and disadvantages of TRUS biopsy compared to TP prostate biopsy are summarised in Table 1, and expanded upon below.

\section{Advantages of TRUS biopsy}

\section{Fast, convenient, and familiar}

TRUS biopsy is a core urological procedure performed by most practising urologists. TRUS biopsy is easily performed in consulting rooms or a minor procedure suite, and takes approximately ten minutes to complete, including the administration of local anaesthesia (LA). Furthermore, the required equipment is widely available in urological centres.

\section{$L A$}

Surprisingly, TRUS biopsy was initially thought to be well tolerated by patients, and analgesia was not routine in the early 1990s. Evidence subsequently emerged to suggest that TRUS biopsy resulted in discomfort and pain in $65 \%$ to $90 \%$ of patients (12-14). Nash and colleagues demonstrated that peri-prostatic nerve blockade (PPNB) with lidocaine significantly improved pain scores (14). PPNB at the time of TRUS biopsy has since become standard practice for most 
Table 1 Pros and cons of TRUS biopsy vs. TP biopsy of the prostate

\begin{tabular}{lll}
\hline Item & TRUS & TP biopsy \\
\hline Pros & Fast & Low risk of urinary sepsis \\
& Convenient & Template approach-stable, assists in cognitive fusion \\
& Familiarity & Access to the anterior prostate \\
& Local anaesthesia & \\
& Lower risk of urinary retention & \\
& Cost-effective & \\
Cons & Antibiotic prophylaxis required due to risk of infection and sepsis & Expensive \\
& Limitations in targeting & Time-consuming \\
& & Usually under general anaesthesia \\
& & Higher rates of urinary retention
\end{tabular}

TRUS, transrectal ultrasound; TP, transperineal.

clinicians (15-17).

Several other analgesic modalities have been trialled to improve the comfort of TRUS biopsy. A review by Lee and colleagues in 2014 confirmed that PPNB had the strongest evidence for optimising comfort during TRUS biopsy. Intrarectal local anaesthetic gel was not found to significantly improve pain outcomes. Propofol, benzodiazepines and opioids may decrease the discomfort experienced during TRUS biopsy, but are associated with additional anaesthetic costs, safety, and longer post-biopsy recovery. These analgesic options remove much of the benefit of TRUS biopsy, which is advantageous in low resource, high volume centres. Peri-operative use of rectal diclofenac was not found to significantly decrease patient discomfort (10).

Short-acting Inhaled anaesthetic agents may be of benefit but require further research (18). Inhaled methoxyflurane (Penthrox) may be an effective adjunct to PPNB while performing TRUS biopsy and is currently being investigated in a multi-centre randomised controlled trial (RCT) in collaboration with the Australian and New Zealand Urogenital and Prostate Trials Group (ANZUP). This Pain-Free TRUS-B Trial has nearly fully accrued 420 participants (19).

\section{Cost effective and accessible}

TRUS biopsy is extremely cost-effective compared to TP biopsy, which requires access to an operating theatre and is usually performed under a general anaesthetic. A brachytherapy grid is frequently used to assist in either cognitive fusion or saturation TP biopsies, and the necessary equipment may be unavailable in many smaller Urology centres. The procedure takes around thirty minutes with extra time required for anaesthesia. TRUS biopsy allows for discharge immediately post-procedure and lends itself to rapid access diagnostic services, whereas TP biopsy requires a day-case admission and increased nursing support. These factors all favour the cost-effectiveness of TRUS biopsy in the public hospital or resource limited healthcare setting.

The economic benefits of TRUS are particularly relevant in the authors' institution. In rural Western Australia, patients travel up to $3,000 \mathrm{~km}$ to access subspecialty care. For these patients, a rapid access approach provides multiparametric MRI (mpMRI) and TRUS biopsy in one day to facilitate fast, cost-effective allocation of healthcare services and rapid cancer diagnosis (20). Patients are able to return home quickly, minimising hospital stay, transport and accommodation costs.

\section{Lower risk of acute urinary retention}

TRUS biopsy has been associated with post-procedural urinary retention, requiring insertion of an indwelling catheter until inflammatory changes resolve. The risk of retention is generally regarded as lower for TRUS than TP biopsy. In a 2017 meta-analysis, Borghesi and colleagues suggested that urinary retention occurs in $0.4-6 \%$ of patients who undergo TRUS biopsy. In comparison, $1.7-11.1 \%$ of patients who received TP biopsy developed 
urinary retention (21). Included in this dataset was a cohort study of 3,000 patients by Pepe and colleagues, who demonstrated that the risk of urinary retention postTP biopsy significantly rose as the number of biopsy cores increased (22).

Tamsulosin has been trialled to minimise the risk of acute urinary retention. Chung and colleagues randomised 88 patients who did not take any previous pharmacotherapy for lower urinary tract symptoms, of which half received tamsulosin from one day pre-TRUS biopsy until seven days post. There was a significant improvement in flow rate, postvoid residual, and retention in the tamsulosin group (23). Despite the small numbers in this study, there may be some benefit to the use of alpha-blockers in the peri-biopsy period.

\section{Disadvantages of TRUS biopsy}

\section{Post-TRUS biopsy infection and sepsis}

The most significant complication of TRUS biopsy is sepsis, which in 2016 was re-defined as "life threatening organ dysfunction caused by a dysregulated host response to infection" (24). Historically, it was defined as "infection plus systemic inflammatory response syndrome" $(25,26)$. The risk of post-operative infection is not surprising, as the TRUS biopsy needle must pass through the rectal wall to access the prostate, inoculating rectal flora. The empirical antibiotic choice at TRUS biopsy is ciprofloxacin. Multiple studies have been performed to reduce the risk of infective complications, to reduce the rectal flora and to treat drugresistant bacteria.

Historically, infection complicated approximately $1 \%$ of patients who underwent TRUS biopsy, but this figure has risen to between $2-4 \%$ in recent years $(27,28)$. The increasing infection rates may be secondary to fluoroquinolone resistance, attributed to recent infection and antibiotic use $(28,29)$. Up to one-quarter of men undergoing prostate biopsy may be colonised with fluoroquinolone-resistant rectal flora (30). Men with significant risk factors for sepsis such as immunocompromise, type 2 diabetes mellitus, recent hospitalisation and COPD (28), could be considered for TP rather than TRUS biopsy.

The duration of pre-biopsy fluoroquinolone prophylaxis appears unrelated to infection risk. Bangash and colleagues performed a prospective analysis of post-biopsy infection rates in 2018 , and suggested that a single dose of ciprofloxacin $500 \mathrm{mg}$ was non-inferior to a three-day course of twice daily ciprofloxacin in over 700 patients (31).

\section{Rectal disinfection}

Rectal disinfection has been trialled in an attempt to reduce the risk of TRUS biopsy related sepsis. In 2014, Pu and colleagues performed a meta-analysis of povidone-iodine preparation with antibiotic prophylaxis, and declared that this significantly reduced the rate of post-biopsy infective complications compared to antibiotics alone (32). However, no adequately powered RCT has confirmed this suggestion. This technique does not remove the need for antibiotic prophylaxis.

\section{Targeted antibiotic prophylaxis}

There may be a clinical role for pre-biopsy rectal swabs and targeted antibiotic prophylaxis. In a 2014 meta-analysis, Roberts and colleagues declared that fluoroquinolone resistant swabs were significantly more likely in patients who had previous exposure to these antibiotics. Infection rates post-TRUS biopsy were significantly higher in people who received pre-operative empirical fluoroquinolones (3.3\%) as compared to targeted antibiotic prophylaxis $(0.3 \%)$ based on rectal flora results (33), although it is possible that many of these patients with fluoroquinolone-resistance rectal isolates were given carbapenems (discussed in more detail below). In a systematic review of nine cohort studies and over 4,500 patients, Cussans and colleagues identified that targeted antibiotic prophylaxis decreased the rate of infective complications from $4.55 \%$ to $0.72 \%$, and sepsis from $2.21 \%$ to $0.48 \%$ (34).

\section{Carbapenem prophylaxis}

The use of carbapenem prophylaxis appears to decrease the rate of infective complications following TRUS biopsy in populations with known fluoroquinolone resistance. In New Zealand, Losco and colleagues performed a prospective audit of ertapenem use in patients deemed to be at high risk of TRUS-related sepsis. These patients had received fluoroquinolones in the 12 months prior, or travelled to South-East Asia within the past 6 months, or were immunocompromised (including diabetes). In 80 high-risk patients who received ertapenem, there were no cases of sepsis compared to a rate of $6.7 \%$ within the cohort who received ciprofloxacin and amoxicillin-clavulanate (35). In several Australian studies, carbapenem use has been associated with a zero-sepsis rate $(31,36)$. Despite the apparent reduction in TRUS biopsy related infection, the use of carbapenems should be minimised where possible 
due to the threat of Enterobacteriaceae carbapenemresistance. This is a major global health concern as it is difficult to treat and is associated with a high-mortality (37). Therefore, carbapenems cannot be recommended for routine prophylaxis.

\section{Diagnostic yield and access to the anterior prostate}

There is a lack of high-quality data comparing the diagnostic yield of TRUS biopsy against TP biopsy. A 2017 meta-analysis from Xue and colleagues reviewed 13 studies comprising over 4,200 patients, and concluded that the overall prostate cancer detection rate between TRUS and TP biopsy was not significantly different (38). No RCT supporting an overall benefit of TP over TRUS biopsy exists. Although mpMRI and fusion technology are suspected to improve sensitivity in small and anterior lesions, further adequately-powered studies are required (39).

Although tumours in the anterior zone are less common than in the peripheral zone, the relative inaccessibility of anterior zone sampling is a clear disadvantage of TRUS compared to TP biopsy. In men with suspicious PSA findings and benign histopathology on TRUS biopsy, TP biopsy should be considered to assess the anterior zone (40). In centres with both TRUS and TP biopsy, patients with suspicious anterior zone lesions on mpMRI should be allocated to a TP biopsy where possible.

\section{mpMRI prostate and targeted biopsy}

The use of pre-biopsy mpMRI may aid in the detection of clinically significant prostate cancer (41). The PRECISION study randomised men to undergo either systematic or targeted biopsies and suggested that targeted biopsy was more sensitive for the detection of clinically significant prostate cancer (42). MRI-FIRST studied 251 men who received mpMRI followed by both targeted and systematic TRUS biopsies. The rate of clinically significant prostate cancer was higher when both targeted and systematic biopsies were performed, and each technique would have missed lesions if performed alone (43). Systematic biopsy ensures the thorough diagnostic assessment of the prostate allowing for the limitations of mpMRI and cognitive fusion using TRUS.

Although mpMRI is typically reliable for identifying higher-grade (ISUP Grade >2) tumours (41), it does have limitations. Up to $16 \%$ of clinically significant lesions can be missed, resulting in a false negative diagnosis (44). Therefore, a decision to abandon biopsy based on negative mpMRI results should be limited to high-volume uroradiological units with extensive operator experience. A significant proportion of the tumours missed on mpMRI will be in the peripheral zone, which is well sampled by TRUS biopsy. Many healthcare systems do not have the resources required to provide routine pre-biopsy mpMRI or fusion software for targeting lesions, thus maintaining the relevance of TRUS biopsy.

\section{Conclusions}

TRUS-guided prostate biopsy has been the standard diagnostic approach for prostate cancer for nearly 30 years and remains a rapid, cost-effective and generally well tolerated technique. TRUS biopsy does have disadvantages, the most significant being the risk of infective complications. With the careful selection of patients and sensible antibiotic use however, these complications can be minimised.

In many cases, such as in patients with risk factors for sepsis and with anterior prostate lesions, TP biopsy should be used in preference to TRUS. However, TRUS biopsy will likely remain a valuable tool in low resource and highvolume centres for some time.

\section{Acknowledgments}

Funding: None.

\section{Footnote}

Provenance and Peer Review: This article was commissioned by the Guest Editor (Shomik Sengupta) for the series "Surgery for Urologic Cancers" published in Translational Andrology and Urology. The article has undergone external peer review.

Conflicts of Interest: Both authors have completed the ICMJE uniform disclosure form (available at http://dx.doi. org/10.21037/tau.2019.09.37). The series "Surgery for Urologic Cancers" was commissioned by the editorial office without any funding or sponsorship. The authors have no other conflicts of interest to declare.

Ethical Statement: The authors are accountable for all aspects of the work in ensuring that questions related to the accuracy or integrity of any part of the work are 
appropriately investigated and resolved.

Open Access Statement: This is an Open Access article distributed in accordance with the Creative Commons Attribution-NonCommercial-NoDerivs 4.0 International License (CC BY-NC-ND 4.0), which permits the noncommercial replication and distribution of the article with the strict proviso that no changes or edits are made and the original work is properly cited (including links to both the formal publication through the relevant DOI and the license). See: https://creativecommons.org/licenses/by-nc-nd/4.0/.

\section{References}

1. Astraldi A. Diagnosis of cancer of the prostate; biopsy by rectal route. Urol Cutaneous Rev 1937;41:421-7.

2. Spirnak JP, Resnick MI. Transrectal ultrasonography. Urology 1984;23:461-7.

3. Hodge KK, McNeal JE, Stamey TA. Ultrasound Guided Transrectal Core Biopsies of the Palpably Abnormal Prostate. J Urol 1989;142:66-70.

4. Hodge KK, McNeal JE, Terris MK, et al. Random Systematic Versus Directed Ultrasound Guided Transrectal Core Biopsies of the Prostate. J Urol 1989;142:71-4.

5. Dietrick DD, McNeal JE, Stamey TA. Core cancer length in ultrasound-guided systematic sextant biopsies: a preoperative evaluation of prostate cancer volume. Urology 1995;45:987-92.

6. Stamey TA. Making the most out of six systematic sextant biopsies. Urology 1995;45:2-12.

7. Eskew LA, Bare RL, McCullough DL. Systematic 5 Region Prostate Biopsy is Superior to Sextant Method for Diagnosing Carcinoma of the Prostate. J Urol 1997;157:199-202; discussion 202-3.

8. Levine MA, Ittman M, Melamed J, et al. Two Consecutive Sets Of Transrectal Ultrasound Guided Sextant Biopsies Of The Prostate For The Detection Of Prostate Cancer. J Urol 1998;159:471-5; discussion 475-6.

9. Presti JC, Chang JJ, Bhargava V, et al. The Optimal Systematic Prostate Biopsy Scheme Should Include 8 Rather than 6 Biopsies: Results of a Prospective Clinical Trial. J Urol 2000;163:163-6; discussion 166-7.

10. Ooi WL, Hawks C, Tan AHH, et al. A randomised controlled trial comparing use of lignocaine periprostatic nerve block alone and combined with diclofenac suppository for patients undergoing transrectal ultrasound (TRUS)-guided prostate biopsy. BJU Int 2014;114 Suppl 1:45-9.
11. Rodriguez LV, Terris MK. Risks and Complications of Transrectal Ultrasound Guided Prostate Needle Biopsy: A Prospective Study and Review of the Literature. J Urol 1998; 160:2115-20.

12. Irani J, Fournier F, Bon D, et al. Patient tolerance of transrectal ultrasound-guided biopsy of the prostate. $\mathrm{Br} \mathrm{J}$ Urol 1997;79:608-10.

13. Collins GN, Lloyd SN, Hehir M, et al. Multiple Transrectal Ultrasound-Guided Prostatic BiopsiesTrue Morbidity and Patient Acceptance. Br J Urol 1993;71:460-3.

14. Nash PA, Bruce JE, Indudhara R, et al. Transrectal Ultrasound Guided Prostatic Nerve Blockade Eases Systematic Needle Biopsy of the Prostate. J Urol 1996;155:607-9.

15. Hiros M, Selimovic M, Spahovic H, et al. Transrectal Ultrasound-Guided Prostate Biopsy, Periprostatic Local Anesthesia and Pain Tolerance. Bosn J Basic Med Sci. 2010;10:68-72.

16. Kahriman G, Donmez H, Mavili E, et al. Transrectal ultrasound guided multi-core prostate biopsy: pain control: results of 106 patients. J Clin Ultrasound 2011;39:270-3.

17. Nambirajan T, Woolsey S, Mahendra V, et al. Efficacy and safety peri-prostatic local anaesthetic injection in transrectal biopsy of the prostrate: a prospective randomised study. Surgeon 2004;2:221-4.

18. Lee C, Woo HH. Current methods of analgesia for transrectal ultrasonography (TRUS)-guided prostate biopsy - a systematic review. BJU Int 2014;113 Suppl 2:48-56.

19. Grummet J, Davis I, Martin A, et al. Randomised Phase 3 Double-Blind Placebo-Controlled Trial of Methoxyflurane with Periprostatic Local Anaesthesia to Reduce the Discomfort of Transrectal Ultrasound-Guided Prostate Biopsy (Pain-Free TRUS B): ANZUP 1501. Asia Pac J Clin Oncol 2015;11:148-9.

20. McCombie SP, Hawks C, Emery JD, et al. A 'One Stop' Prostate Clinic for rural and remote men: a report on the first 200 patients. BJU Int 2015;116:11-7.

21. Borghesi M, Ahmed H, Nam R, et al. Complications After Systematic, Random, and Image-guided Prostate Biopsy. Eur Urol 2017;71:353-65.

22. Pepe P, Aragona F. Morbidity After Transperineal Prostate Biopsy in 3000 Patients Undergoing 12 vs 18 vs More Than 24 Needle Cores. Urology 2013;81:1142-6.

23. Chung SJ, Jung SI, Ryu JW, et al. The preventive effect of tamsulosin on voiding dysfunction after prostate biopsy: a prospective, open-label, observational study. Int Urol 
Nephrol 2015;47:711-5.

24. Rhodes A, Evans LE, Alhazzani W, et al. Surviving Sepsis Campaign: International Guidelines for Management of Sepsis and Septic Shock: 2016. Intensive Care Med 2017;43:304-77.

25. Bone RC, Balk RA, Cerra FB, et al. Definitions for sepsis and organ failure and guidelines for the use of innovative therapies in sepsis. The ACCP/SCCM Consensus Conference Committee. American College of Chest Physicians/Society of Critical Care Medicine. Chest 1992;101:1644-55.

26. Levy MM, Fink MP, Marshall JC, et al. 2001 SCCM/ ESICM/ACCP/ATS/SIS International Sepsis Definitions Conference. Intensive Care Med 2003;29:530-8.

27. Bruyère F, Malavaud S, Bertrand $P$, et al. Prosbiotate: A Multicenter, Prospective Analysis of Infectious Complications after Prostate Biopsy. J Urol 2015;193:145-50.

28. Carignan A, Roussy JF, Lapointe V, et al. Increasing Risk of Infectious Complications After Transrectal UltrasoundGuided Prostate Biopsies: Time to Reassess Antimicrobial Prophylaxis? Eur Urol 2012;62:453-9.

29. Loeb S, Carter HB, Berndt SI, et al. Complications After Prostate Biopsy: Data From SEER-Medicare. J Urol 2011;186:1830-4.

30. Cohen JE, Landis P, Trock BJ, et al. Fluoroquinolone Resistance in the Rectal Carriage of Men in an Active Surveillance Cohort: Longitudinal Analysis. J Urol 2015;193:552-6.

31. Bangash HK, Hawks C, McCombie SP, et al. Transrectal prostate biopsy sepsis rate following reduced quinolone antibiotic prophylaxis from six doses to single dose. ANZ J Surg 2018. [Epub ahead of print].

32. Pu C, Bai Y, Yuan H, et al. Reducing the risk of infection for transrectal prostate biopsy with povidone-iodine: a systematic review and meta-analysis. Int Urol Nephrol 2014;46:1691-8.

33. Roberts MJ, Williamson DA, Hadway P, et al. Baseline prevalence of antimicrobial resistance and subsequent infection following prostate biopsy using empirical or altered prophylaxis: A bias-adjusted meta-analysis. Int J Antimicrob Agents 2014;43:301-9.

34. Cussans A, Somani BK, Basarab A, et al. The role of targeted prophylactic antimicrobial therapy before transrectal ultrasonography-guided prostate biopsy in reducing infection rates: a systematic review. BJU Int
2016;117:725-31.

35. Losco G, Studd R, Blackmore T. Ertapenem prophylaxis reduces sepsis after transrectal biopsy of the prostate. BJU Int 2014;113:69-72.

36. Leahy OR, O' Reilly M, Dyer DR, et al. Transrectal ultrasound-guided biopsy sepsis and the rise in carbapenem antibiotic use. ANZ J Surg 2015;85:931-5.

37. Sheu CC, Chang Y'T, Lin SY, et al. Infections Caused by Carbapenem-Resistant Enterobacteriaceae: An Update on Therapeutic Options. Front Microbiol 2019;10:80.

38. Xue J, Qin Z, Cai H, et al. Comparison between transrectal and transperineal prostate biopsy for detection of prostate cancer: a meta-analysis and trial sequential analysis. Oncotarget 2017;8:23322-36.

39. Wegelin O, Exterkate L, van der Leest M, et al. The FUTURE Trial: A Multicenter Randomised Controlled Trial on Target Biopsy Techniques Based on Magnetic Resonance Imaging in the Diagnosis of Prostate Cancer in Patients with Prior Negative Biopsies. Eur Urol 2019;75:582-90.

40. Mabjeesh NJ, Lidawi G, Chen J, et al. High detection rate of significant prostate tumours in anterior zones using transperineal ultrasound-guided template saturation biopsy. BJU Int 2012;110:993-7.

41. Bratan F, Niaf E, Melodelima C, et al. Influence of imaging and histological factors on prostate cancer detection and localisation on multiparametric MRI: a prospective study. Eur Radiol 2013;23:2019-29.

42. Kasivisvanathan V, Rannikko AS, Borghi M, et al. MRITargeted or Standard Biopsy for Prostate-Cancer Diagnosis. N Engl J Med 2018;378:1767-77.

43. Rouvière $\mathrm{O}$, Puech $\mathrm{P}$, Renard-Penna R, et al. Use of prostate systematic and targeted biopsy on the basis of multiparametric MRI in biopsy-naive patients (MRIFIRST): a prospective, multicentre, paired diagnostic study. Lancet Oncol 2019;20:100-9.

44. Borofsky S, George AK, Gaur S, et al. What Are We Missing? False-Negative Cancers at Multiparametric MR Imaging of the Prostate. Radiology 2018;286:186-95.

Cite this article as: Moe A, Hayne D. Transrectal ultrasound biopsy of the prostate: does it still have a role in prostate cancer diagnosis? Transl Androl Urol 2020;9(6):3018-3024. doi: 10.21037/ tau.2019.09.37 